Classification

Physics Abstracts

$61.70 \mathrm{~J}-61.70 \mathrm{~N}-68.48-64.75$

\title{
Dislocations extrinsèques, ségrégation intergranulaire et mobilité des joints de grains
}

\author{
S. Lartigue, O. Khalfallah et L. Priester \\ Laboratoire de Métallurgie Physique (*), Université de Paris-Sud, 91405 Orsay Cedex, France
}

(Reçu le 19 février 1982, révisé le 28 juin 1982, accepté lè 2 juillet 1982)

\begin{abstract}
Résumé. - La présence de dislocations extrinsèques dans les joints de grains de divers alliages ferreux cubiques centrés, a été étudiée en fonction de la ségrégation et de la précipitation intergranulaire. Les auteurs émettent l'hypothèse d'une ségrégation " active " susceptible de freiner ou bloquer les réactions des dislocations extrinsèques dans le joint, réactions conduisant à leur annihilation. Deux facteurs conditionnent cette ségrégation " active » :

- une forte affinité chimique entre éléments ségrégés,

- un facteur de taille favorable entre ces éléments et le fer.

Une tentative d'interprétation des conséquences des interactions dislocations-impuretés sur la mobilité des joints de grains est également avancée.
\end{abstract}

\begin{abstract}
The occurrence of extrinsic dislocations in grain boundaries of various body centred ferrous alloys has been studied in relation with the intergranular segregation and precipitation. The authors pointed out that an « active " segregation is allowed to restrain or to impede the reactions of extrinsic dislocations in the boundary, these reactions involving their annihilation. Two parameters are necessary :

- a strong affinity between the segregated species,

- a favourable size effect between the species and iron.

The authors tried out to interpret the consequences of the interactions between dislocations and impurities on the mobility of grain boundaries.
\end{abstract}

1. Introduction - Dans les matériaux polycristallins, les défauts de structure des joints de grains jouent un rôle souvent plus important que leur structure sur certaines propriétés des interfaces, en particulier sur leurs propriétés mécaniques et sur leur migration. Ces défauts, ponctuels et linéaires, sont d'ailleurs fonction de la structure d'équilibre du joint et/ou de son énergie intergranulaire [1] et il existe, comme dans le cristal lui-même, une interaction entre les différents types de défauts.

Cette étude se propose d'établir une relation entre la nature, l'état (élémentaire ou précipité) et la répartition des impuretés ségrégées dans les joints de grains d'alliages de fer cubiques centrés et la présence de défauts linéaires intergranulaires ou "dislocations extrinsèques ".

Elle aborde également les conséquences des interactions éventuelles entre défauts sur la mobilité des interfaces.

(*) Associé au C.N.R.S. LA 177.
2. Structure et défauts de structure des joints de grains. - L'étude de la structure et des défauts de structure des joints de grains des polycristaux réels s'effectue principalement en microscopie électronique. Elle s'appuie sur le formalisme de Bollmann [2,3] bien adapté aux systèmes cristallins à haute symétrie (cubique, hexagonale).

Les joints réels sont définis par un axe de désorientation $R$ et un angle $\theta$ autour de cet axe et par l'orientation du plan du joint dans l'un quelconque des deux grains adjacents; à partir de ces paramètres et du formalisme de Bollman, on distingue alors deux types de joints :

- les joints spéciaux proches d'une position de coïncidence définie par un indice $\Sigma$. Cependant la notion de spécialité n'est pas absolue, elle dépend en particulier du projet de l'étude et de certaines conditions expérimentales [1]. Théoriquement, tout joint peut être décrit par un arrangement périodique de dislocations intrinsèques, en particulier, tout écart $\Delta \theta$, à la position de coïncidence est accommodé 


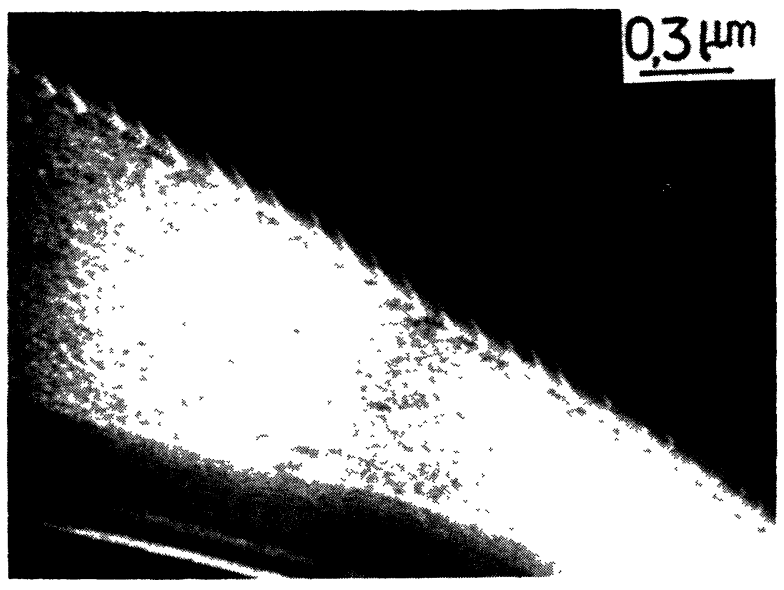

Fig. 1. - Joint de grains dans un alliage Fe-1 \% Mo présentant un réseau de dislocations intrinsèques périodiques perturbé par quelques dislocations extrinsèques de contraste nettement plus marqué.

[Grain boundary in an $\mathrm{Fe}-1 \%$ Mo alloy with a periodic set of intrinsic dislocations locally disturbed by extrinsic dislocations exhibiting a strong contrast.]

par l'apparition d'un réseau de dislocations secondaires (primaires si $\Sigma=1$ ) dont les vecteurs de Burgers sont les vecteurs de base du réseau D.S.C. [2]. Mais, ces dislocations ne sont pas toujours visibles, en particulier dans les joints des polycristaux réels, bien qu'elles soient géométriquement nécessaires. Le critère de spécialité n'est donc pas obligatoirement lié à la visibilité des dislocations intrinsèques. L'écart $\Delta \theta$ pour un $\Sigma$ donné a été calculé sur la base des travaux de Warrington et Boon [4] ; compte tenu de la précision expérimentale sur $\theta\left(\Delta \theta \leqq 2^{\circ}\right)$ limitée, entre autres,

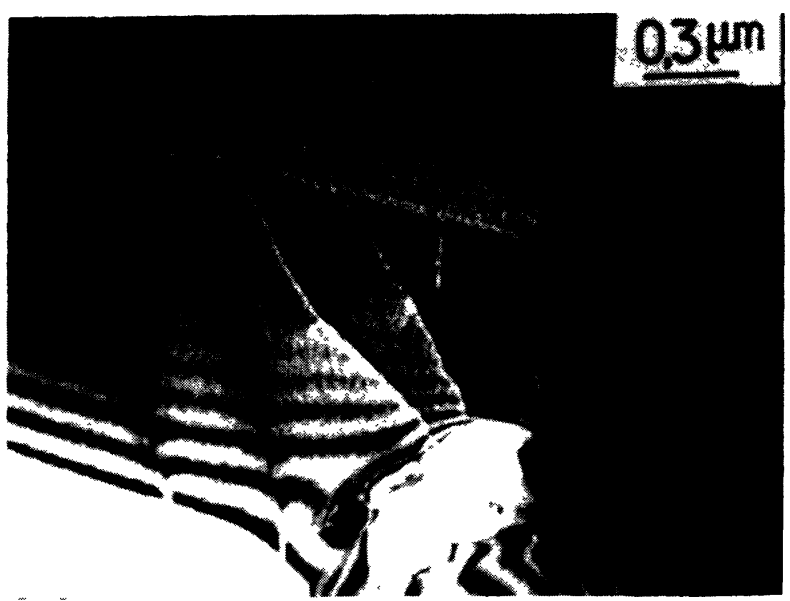

Fig. 2. - Les dislocations de matrice (A) perdent leur contraste oscillant lorsqu'elles pénètrent dans le joint de grains d'un alliage $\mathrm{Fe}-9 \% \mathrm{Cr}$ pour former des dislocations extrinsèques $(B)$.

[The matrix dislocations (A) loose their oscillating contrast when they impinge on grain boundaries creating extrinsic dislocations (B) (Fe-9\% $\mathrm{Cr}$ alloy).] par le ferromagnétisme des alliages étudiés seuls les joints de $\Sigma \leqq 17$ peuvent être considérés, sans ambiguïté, comme joints spéciaux $[5,6]\left({ }^{1}\right)$.

- les joints généraux éloignés de toute position de coïncidence.

La structure d'équilibre des joints de grains peut être perturbée par des "dislocations extrinsèques " qui, contrairement aux dislocations primaires et secondaires, sont des défauts linéaires isolés conservant un champ de contrainte élastique à grande distance (Fig. 1). Les vecteurs de Burgers de ces dislocations sont également des vecteurs du réseau D.S.C.; ils dépendent de l'origine des dislocations et du type du joint. Les dislocations extrinsèques résultent le plus souvent de l'interaction des dislocations de matrice avec le joint (Fig. 2). On distingue 2 types d'interactions (Fig. 3) :

- soit la dislocation de matrice traverse le joint ce qui peut se produire si les plans de glissement dans les deux grains contigus ont un axe de zone commun dans ou proche du plan du joint [7]; elle laisse alors dans

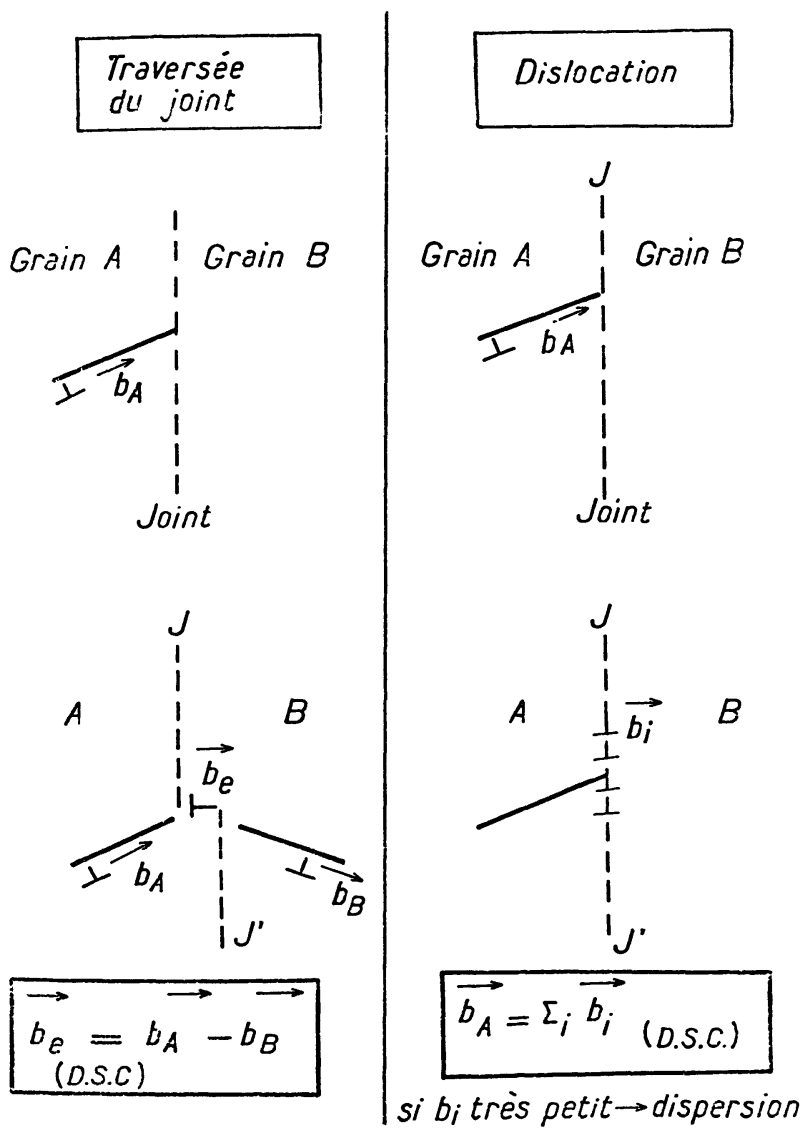

Fig. 3. - Schéma des interactions possibles entre une dislocation de matrice et un joint de grain.

[Models of possible interactions between a matrix dislocation and a grain boundary.]

( $\left.{ }^{1}\right)$ Sont également classés comme joints spéciaux les joints de $\Sigma$ plus élevés et dont $R$ a des indices simples décrits par le modèle de coïncidence d'axe (C.A.D.) $[4,5]$. 
l'interface une dislocation dont le vecteur de Burgers est un vecteur du réseau D.S.C.,

- soit il y a transfert total de la contrainte à l'interface. Dans ce cas, les dislocations peuvent conserver le vecteur de Burgers de la matrice ou se dissocier en plusieurs dislocations dont $b=\mathbf{b}_{\text {D.s.C. }}$ avec la condition :

$$
b_{\text {matrice }}=\sum_{i} b_{i_{\text {D.s.c. }}} .
$$

Quel que soit le mécanisme, une dislocation sera visible dans les joints spéciaux où les $b_{\text {D.s.c. }}$ ne sont pas négligeables $[8,9]$.

Dans les deux hypothèses d'interactions, les conditions pour lesquelles on peut observer des dislocations extrinsèques, c'est-à-dire les conditions de stabilité de ces dislocations, dépendent de l'histoire thermomécanique et chimique du matériau et font actuellement l'objet de controverses.

3. Dislocations extrinsèques. Propriétés du joint. But du travail. - Certains auteurs ont montré que les dislocations extrinsèques introduites par déformation plastique à température ambiante disparaissent extrêmement rapidement sous l'effet d'un recuit modéré $[10,11,12]$. Ce comportement est interprété par un étalement du cœur de la dislocation [13] ou, ce qui est formellement équivalent, par une dissociation en un grand nombre de dislocations dont les vecteurs de Burgers sont infiniment petits [14]. Par ce processus, les dislocations extrinsèques s'incorporent à la structure intrinsèque du joint en la modifiant. Les propriétés du joint semblent dépendre de ces mécanismes, qu'il s'agisse de la recristallisation où les joints peuvent migrer par les mécanismes associés d'absorption de dislocations et de formation de marches [15] ou de la déformation plastique qui nécessite la traversée des joints de grains par les dislocations de matrice. Dans ce dernier cas, si les dislocations extrinsèques formées au début de la déformation sont stables dans le joint, elles s'opposent au passage ultérieur des dislocations, le joint est dit " dur » ; si au contraire, la dispersion peut avoir lieu, la déformation se poursuit, le joint est dit « mou » [16]. Une autre conséquence importante de la stabilisation des dislocations extrinsèques est leur possibilité d'être sites de nucléation de précipités. Varin, comparant les cinétiques du processus de dispersion et la précipitation intergranulaire conclut à l'impossibilité d'une germination sur les lignes de dislocations extrinsèques [17]. Or, des observations effectuées sur divers matériaux révèlent l'existence de précipités intergranulaires alignés selon des directions préférentielles, observations que les auteurs interprètent comme le résultat d'une association entre les défauts linéaires du joint et la précipitation $[6,18,19$, 20, 21].

Dans un article antérieur, nous avions émis l'hypothèse que seule, une ségrégation intergranulaire dite « active » d'éléments présentant une forte affinité chimique pouvait freiner ou bloquer les processus de dispersion et que, dans ce cas, une précipitation sur les lignes de dislocations extrinsèques pourrait intervenir [6].

L'objet du présent travail est :

- de compléter, dans un but statistique, les résultats obtenus précédemment sur le fer et les alliages $\mathrm{Fe}-4 \% \mathrm{Ni}, \mathrm{Fe}-9 \% \mathrm{Cr}$,

- de confirmer ou d'infirmer par l'étude d'un autre alliage de fer, judicieusement choisi (Fe-1\% Mo), l'hypothèse de la ségrégation active,

- de préciser le lien entre les dislocations extrinsèques et la précipitation,

- de dégager les conséquences de ces phénomènes sur la mobilité des joints de grains.

4. Résultats et interprétations. - 4.1 DiSLOCATIONS EXTRINSÈQUES. SÉGRÉGATION ET PRÉCIPITATION INTERGRANULAIRE. - Le fer et les alliages de fer cubiques centrés de haute pureté contiennent uniquement l'impureté carbone à des teneurs faibles et variables (cf. tableau I). Ils subissent un traitement de recristallisation en phase $\alpha$ à une température pour laquelle les processus de dispersion des dislocations extrinsèques devraient avoir lieu [9, 10].

Les résultats des études en microscopie électronique rassemblés dans le tableau I montrent que, parmi les quatre matériaux étudiés, seul l'alliage $\mathrm{Fe}-9 \% \mathrm{Cr}$ présente des dislocations extrinsèques et ce, essentiellement, dans les joints généraux $\left({ }^{2}\right)$. Le nombre de joints contenant ces dislocations et la densité de celles-ci dépendent de la teneur globale en carbone et de la précipitation. La présence de ces défauts nécessite une nette sursaturation en carbone ( $90 \mathrm{ppm})$, la solubilité limite théorique de cet élément à $780^{\circ} \mathrm{C}$ dans l'alliage Fe-9 \% chrome étant de 15 ppm [22]. Au-delà, la quantité de défauts commence par augmenter, puis pour des teneurs globales voisines de $150 \mathrm{ppm}$ des micro-précipités intergranulaires se forment parallèlement aux lignes de dislocations qui, simultanément, ne sont plus visibles dans les régions de précipitation (Figs. 4-5). Lorsque la teneur en carbone atteint 250 ppm environ, de gros carbures $\mathrm{M}_{23} \mathrm{C}_{6}$ apparaissent dans l'interface alors totalement dépourvue de dislocations (Fig. 6).

Notons que pour l'alliage Fe-Mo, des précipités de $\mathrm{Mo}_{2} \mathrm{C}$ inter- et intragranulaires de taille relativement importante se forment dès une teneur en carbone voisine de $10^{-4}$ (Fig. 7).

La différence de comportement des alliages $\mathrm{Fe}-9 \%$ $\mathrm{Cr}$ et $\mathrm{Fe}-1 \% \mathrm{Mo}$, bien qu'il existe une interaction chimique forte entre les deux éléments substitutionnels et le carbone, nous amène à revoir les paramètres conditionnant l'activité d'une ségrégation vis-à-vis

$\left({ }^{2}\right)$ Signalons que quelques rares joints spéciaux, dans lesquels les réseaux de dislocations intrinsèques sont souvent visibles, présentent également des défauts hors d'équilibres (cf. chap. 2). 
Tableau I. - Présence de dislocations extrinsèques en relation avec l'état de ségrégation ou de précipitation du carbone dans les interfaces de divers alliages de fer. Les possibilités de ségrégation du carbone sont déduites de la considération des coefficients d'enrichissement $\beta$ [29] et de résultats antérieurs [22-30].

[Occurrence of extrinsic dislocations in relation with the segregation and the precipitation of carbon in the grain boundaries of various iron alloys. The possibilities of carbon segregation are deduced from the consideration of the enrichment factor $\beta$ [29] and from previous results [22-30].]

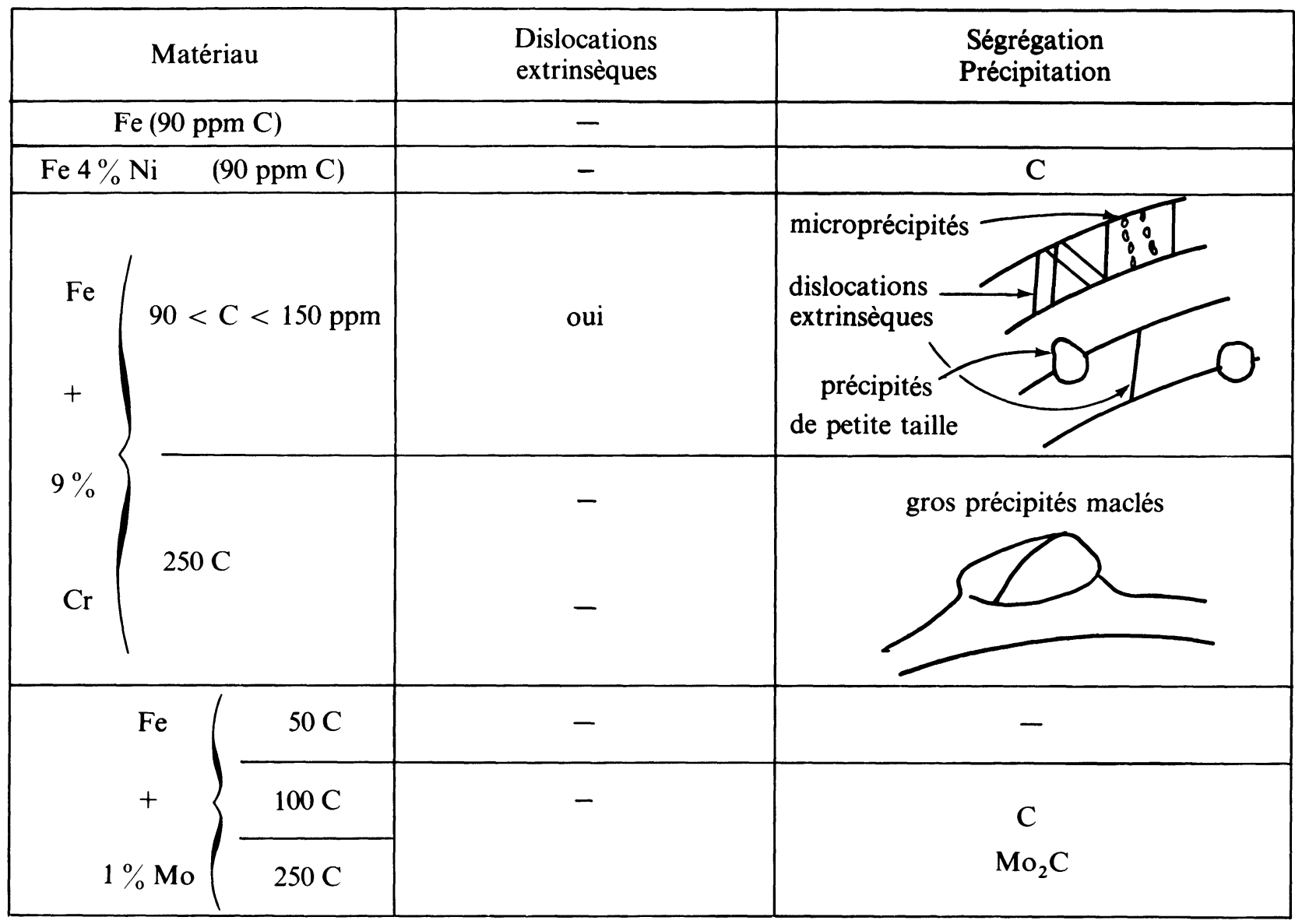

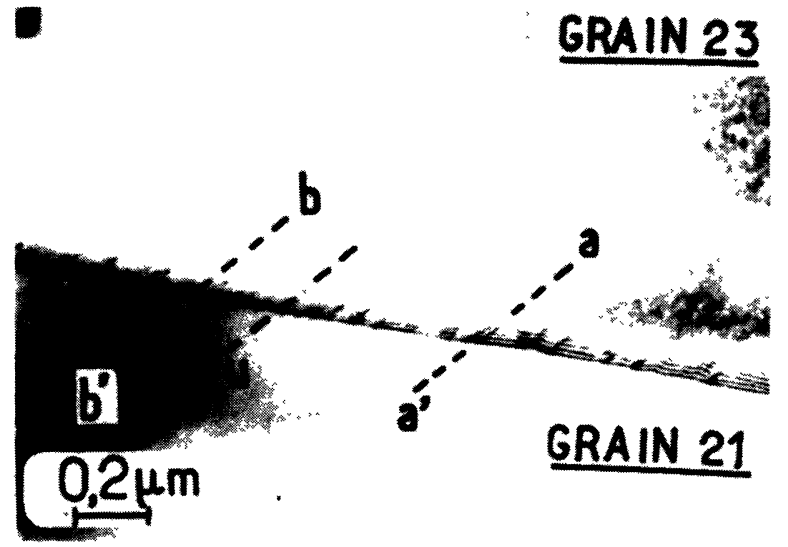

Fig. 4. - Présence simultanée de dislocations extrinsèques $\left(\mathrm{aa}^{\prime}\right)$ et de microprécipités alignés parallèlement à celles-ci $\left(\mathrm{bb}^{\prime}\right)$ dans un alliage fer-chrome $90 \mathrm{C}$.

[Simultaneous occurrence of extrinsic dislocations $\left(\mathrm{aa}^{\prime}\right)$ and parallel arrays of microprecipitates $\left(\mathrm{bb}^{\prime}\right)$ in a $\mathrm{Fe}-9 \% \mathrm{Cr}$ $90 \mathrm{C}$ alloy.]

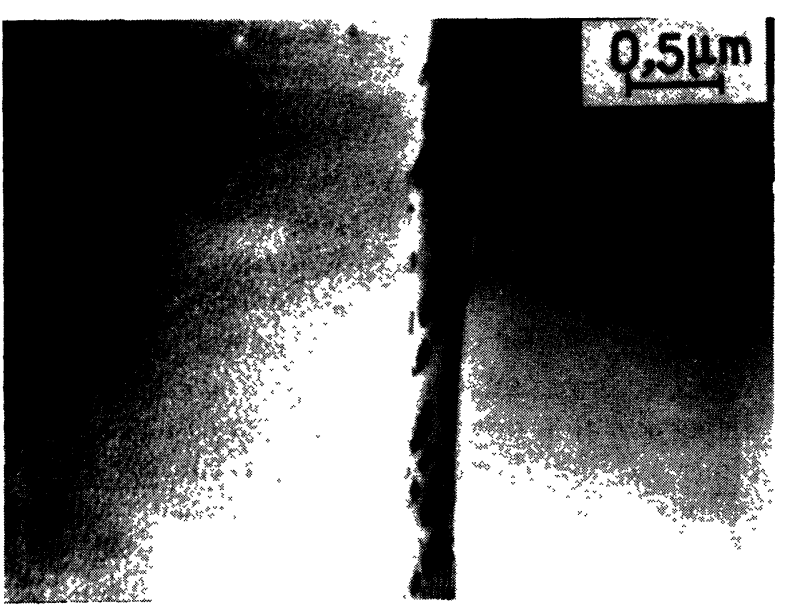

Fig. 5. - L'existence d'une direction préférentielle de précipitation dans l'interface est nettement mise en évidence sur ce joint d'alliage $\mathrm{Fe}-9 \% \mathrm{Cr}$.

[Precipitates elongated on a preferential direction can be observed in a grain boundary of $\mathrm{Fe}-9 \% \mathrm{Cr}$ alloy.] 


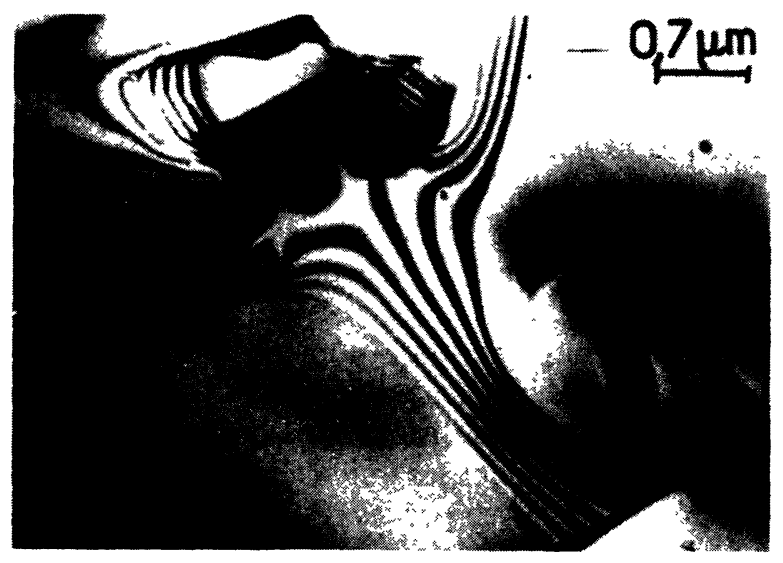

Fig. 6. - Absence de dislocations extrinsèques dans un joint de grains contenant un gros carbure macle $\mathrm{M}_{23} \mathrm{C}_{6}$.

[Presence of a twinned carbide $\mathrm{M}_{23} \mathrm{C}_{6}$ in a grain boundary without extrinsic dislocation.]

de la stabilité des dislocations extrinsèques [6]. Il est important de noter, de ce point de vue, que la précipitation apparait comme un phénomène défavorable.

Les résultats de la présente étude nous conduisent, toujours en accord avec nos hypothèses précédentes, à penser que c'est sous la forme d'amas X-C (X élément substitutionnel dans le fer) que les éléments ségrégés peuvent ancrer les dislocations extrinsèques. Mais, cette formation d'amas nécessite deux conditions : non seulement un facteur chimique favorable [6] mais, conjointement, un facteur élastique favorable. Le rayon atomique de l'élément $X$ doit être inférieur, ou très peu supérieur, à celui du fer pour permettre aux atomes $\mathrm{X}$ d'entourer le carbone, ce qui se produira alors préférentiellement dans les régions de distorsion dans l'interface associées aux dislocations hors d'équi-

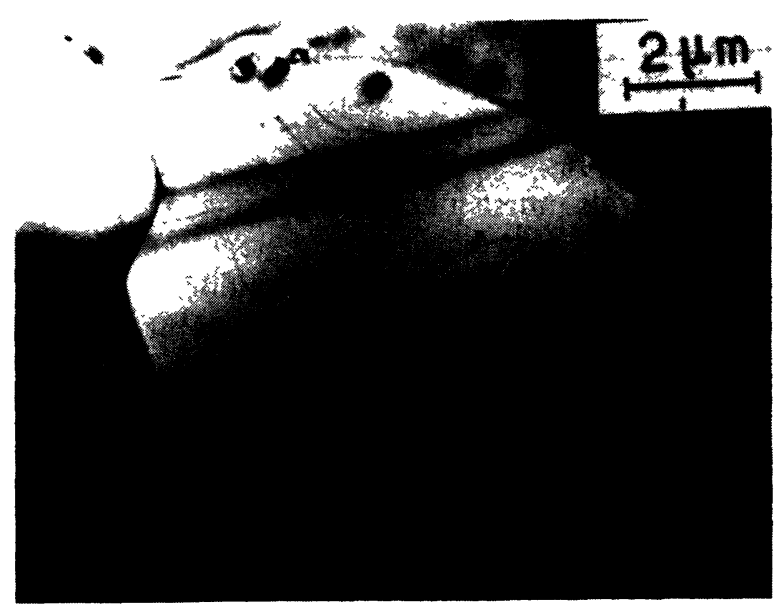

Fig. 7. - Les précipités de $\mathrm{Mo}_{2} \mathrm{C}$ dans l'alliage Fe-Mo contenant $10^{-4} \mathrm{C}$ se situent aux joints de grains et dans la matrice où ils constituent des alignements.

$\left[\mathrm{Mo}_{2} \mathrm{C}\right.$ precipitates in a $\mathrm{Fe}-1 \% \mathrm{Mo}$ containing $10^{-4}$ can be seen in the grain boundaries and in the matrix where they form arrays.] libre (extrinsèques). Ces deux conditions sont réunies uniquement pour l'alliage fer-chrome $\left({ }^{3}\right)$ (Tableau II).

Tableau II. - Ordre de grandeur des facteurs chimique et élastique de ségrégation " active " pour les alliages Fe-X-I :

- facteur chimique :

$$
\Delta \Sigma=\Sigma_{\mathrm{X}-\mathrm{I}}-\Sigma_{\mathrm{Fe}-\mathrm{I}}
$$

si $\Delta \Sigma<0 \rightarrow$ formation d'amas X-I ( $\Sigma$ : énergie de liaison entre atomes proches voisins)

- facteur élastique :

$$
\frac{r_{\mathrm{Fe}}-r_{\mathrm{X}}}{r_{\mathrm{Fe}}}>0 \text { ou } \lesssim 0
$$

[Some approaching values of chemical and elastic parameters of « active» segregation in alloys Fe-X-I'. - chemical parameter :

$$
\Delta \Sigma=\Sigma_{\mathrm{X}-\mathrm{I}}-\Sigma_{\mathrm{Fe}-\mathrm{I}}
$$

$\Delta \Sigma<0 \rightarrow$ clusters X-I $(\Sigma$ : bond energy between neighbouring atoms)

- elastic parameter

$$
\begin{array}{lllc} 
& \multicolumn{2}{c}{\frac{r_{\mathrm{Fe}}-r_{\mathrm{X}}}{r_{\mathrm{Fe}}}>0 \text { or }} & \multicolumn{1}{c}{0 .]} \\
\mathrm{I} & \mathrm{X} & \mathrm{kJ} / \mathrm{mole} & \frac{r_{\mathrm{Fe}}-r_{\mathrm{X}}}{r_{\mathrm{Fe}}}[24] \\
- & - & - & - \\
\mathrm{C} & \mathrm{Cr} & -12,52 & -1,18 \\
\mathrm{~N} & \mathrm{Ni} & +7,56 & +1,97 \\
& \mathrm{Mo} & -5,59 & -10,24
\end{array}
$$

Le fait que le carbone seul ne semble pas pouvoir stabiliser les dislocations extrinsèques peut s'expliquer par la nécessité, résultant du champ de contrainte de ces dislocations, d'une dimension de l'atmosphère d'atomes ségrégés (amas) supérieure à celle d'une atmosphère de Cottrell dans la matrice. Cependant, le rayon de l'amas ne doit pas dépasser la valeur critique correspondant à la barrière de germination car on doit alors s'attendre à une déstabilisation de la dislocation. On constate en effet, la disparition des segments de dislocations entre les microprécipités dont la forme souvent allongée, parallèlement à la ligne du défaut, peut s'expliquer par la diffusion préférentielle des atomes de soluté le long du coeur de la dislocation (Fig. 8) [25]. Ces microprécipités servent

$\left({ }^{3}\right)$ Les données énergétiques du tableau II concernent le fer C.F.C. et l'azote dans le cas de l'alliage Fe-Mo, mais les valeurs propres aux divers alliages cubiques centrés $\mathrm{Fe}-\mathrm{X}-\mathrm{C}$ conservent le même signe et, avec des fortes probabilités, le même ordre :

$$
\Sigma_{\mathrm{Cr}-\mathrm{C}}<\Sigma_{\mathrm{Mo}-\mathrm{C}}<\Sigma_{\mathrm{Ni}-\mathrm{C}} .
$$




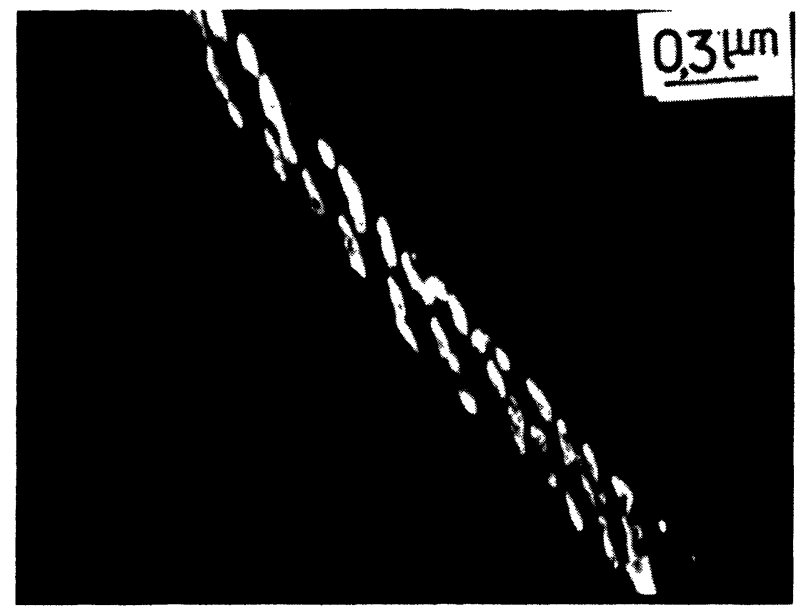

Fig. 8. - Mise en évidence de la forme allongée des précipités $\mathrm{M}_{23} \mathrm{C}_{6}$ intergranulaires revélée sur une micrographie électronique en fond noir.

[Dark field micrograph showing the elongated form of intergranular microprecipitates $\mathrm{M}_{23} \mathrm{C}_{6}$.]

ensuite le puits efficace pour le chrome et le carbone; formation de gros carbures $\mathrm{M}_{23} \mathrm{C}_{6}$ [26].

Dans cette étape de la précipitation intergranulaire, nécessitant une sursaturation relativement importante en carbone et dépendant vraisemblablement de la température et de la durée du traitement thermique, aucune relation n'est mise en évidence entre précipités et défauts. Au contraire, lorsque la précipitation est limitée par la teneur globale en carbone et/ou par sa cinétique, le phénomène apparaît associé aux défauts de structure de l'interface. Cette constatation est en accord avec celles d'autres auteurs qui ont montré que l'effet du joint ne contrôle la précipitation intergranulaire que pour de faibles sursaturations [27, 28].

Les résultats contradictoires des études récentes dans ce domaine peuvent s'interpréter par la non prise en compte des étapes de ségrégation et par la méconnaissance de la cinétique de précipitation intergranulaire. Ainsi, Varin [17] observe la présence de carbures $\mathrm{M}_{23} \mathrm{C}_{6}$ dans les joints généraux d'aciers inoxydables sans relations avec les dislocations isolées dans ces joints qui disparaissent, selon lui, avant que la précipitation ne débute. Cependant cet auteur souligne l'importance des cinétiques comparées de " dispersion " et de "précipitation " et évoque la possibilité pour les dislocations des joints spéciaux, d'être sites de nucléation. Sur le même type d'alliages, Adamson et Martin [18] relient la densité des précipités $\mathrm{M}_{23} \mathrm{C}_{6}$ à la densité des dislocations extrinsèques.

A l'appui de nos résultats citons ceux de Butler et Swann [19] qui mettent en évidence l'alignement sur les dislocations de précipités de $\mathrm{MgZn}_{2}$ dans les joints d'alliage $\mathrm{Al}-\mathrm{Mg}-\mathrm{Zn}$ après vieillissement sous contrainte. Les travaux suivis dans ce domaine de Jones, Howell et Ralph sur les aciers inoxydables stabilisés au niobium, montrent d'une part, que la croissance des carbures intergranulaires $\mathrm{NbC}$ se fait par élongation le long des lignes de dislocations [20], d'autre part, que la densité et la répartition de ces carbures est fonction du pourcentage de déformation subie par le polycristal dont résultent la densité et la répartition des défauts intergranulaires [21].

Les dislocations extrinsèques apparaissent bien comme des sites préférentiels de nucléation des précipités; lorsque ceux-ci se développent, les forces d'ancrage diminuent, le phénomène de "dispersion " qui nécessite le déplacement des produits de la réaction peut intervenir et les dislocations hors d'équilibre ne sont plus visibles dans les joints de grains. Cette interprétation est appuyée par les résultats obtenus sur les alliages $\mathrm{Al}-\mathrm{Cu}$ dans lesquels ce sont les joints sans précipité qui présentent des lignes d'accident, selon la terminologie utilisée par Forest [25], cet auteur signale également que ces lignes servent de sites préférentiels de germination.

En conclusion, nos travaux non seulement appuient la possibilité d'un lien entre précipitation intergranulaire et dislocations extrinsèques mais, de plus, précisent dans quelles conditions ce lien peut exister. Dans les alliages ferreux et, vraisemblablement tout au moins dans les alliages des métaux de transition, l'interface doit être le siège d'une ségrégation dite " active ", caractérisée par une forte affinité chimique entre éléments ségrégés et un facteur élastique favorable entre ces derniers et le métal de base. On peut penser que dans les alliages à caractère purement métallique, le facteur élastique devient prépondérant alors que dans les métaux où les éléments de transition ségrègent, le facteur électronique prend une grande importance.

4.2 Dislocations EXTRINSÈQues ET MOBILITÉ DES JOINTS DE GRAINS. - Dans un travail antérieur, nous avions tenté d'interpréter les phénomènes intervenant lors de la recristallisation des alliages en tenant compte d'une certaine similitude entre les effets de la ségrégation intergranulaire d'une part sur la stabilité des dislocations extrinsèques, d'autre part sur la mobilité des joints de grains [6].

Dans la présente étude, nous nous efforçons de préciser le raisonnement précédent en considérant non seulement les microstructures observées à l'échelle optique (taille des grains, profils équilibrés ou non des interfaces) mais le pourcentage de joints spéciaux déterminé par une étude statistique en microscopie électronique.

A partir de ces deux ensembles de résultats macroscopiques, on peut tenter de comprendre l'influence des phénomènes décrits ci-dessus (cf. paragraphe 4.1) sur la mobilité des interfaces.

Les différents pourcentages de joints spéciaux, établis après calcul de la désorientation sur 50 à 100 joints dans chaque cas, et les microstructures observées parallèlement, sont réunis dans le tableau III. On peut distinguer 2 types de comportements :

- Dans les matériaux les plus purs (fer et $\mathrm{Fe}-1 \% \mathrm{Mo}$ contenant $50 \mathrm{ppm} \mathrm{C)}$ ), la structure se compose de gros 
Tableau III. - Relation entre deux aspects macroscopiques de la recristallisation des alliages de fer : - les microstructures obtenues, - les pourcentages de joints « spéciaux » et "généraux ».

[Relation between two macroscopic aspects of the recrystallization of iron alloys : - the microstructures, - the proportions of « special " and "general » boundaries.]

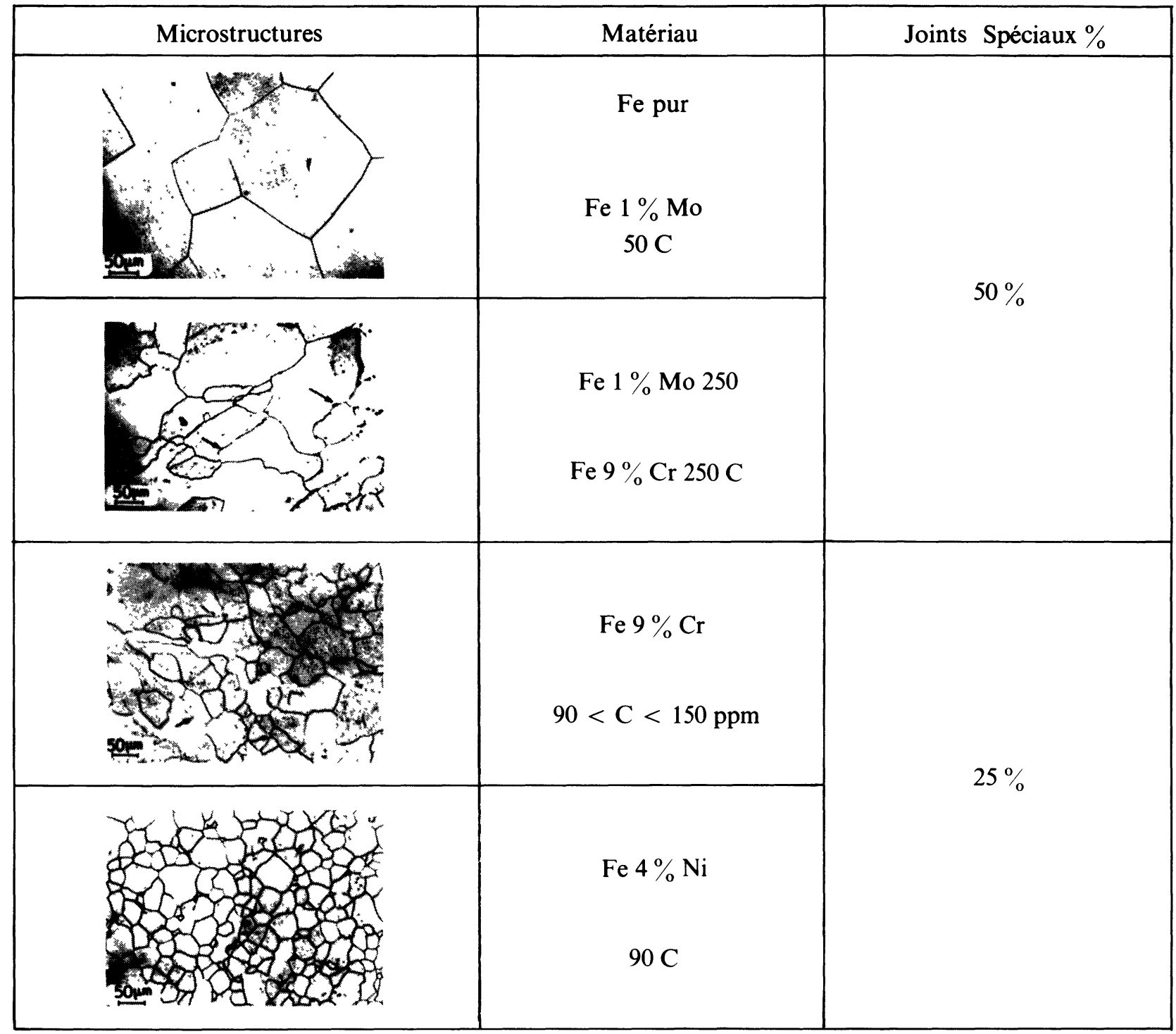

joints équilibrés, dont les dimensions sensiblement identiques sont de l'ordre de $200 \mu \mathrm{m}$. Dans les alliages de teneur élevée en carbone pour lesquels une précipitation intergranulaire intervient, les contours des grains sont perturbés, leurs dimensions varient notoirement mais peuvent atteindre également des ordres de grandeur de $200 \mu \mathrm{m}$.

Dans ces deux cas, on obtient un pourcentage élevé de joints spéciaux (ou de portions de joints), $50 \%$ environ.

A partir de ces résultats, on peut en déduire que les joints de grains ont pu migrer librement pour atteindre leur position d'équilibre " géométrique ». Ceci s'explique pour les matériaux à faible teneur en carbone car la recristallisation primaire n'est à aucun moment freinée par des phénomènes de ségrégation. Pour les teneurs plus élevées en carbone ( $\mathrm{Fe}-\mathrm{Cr}$, $\mathrm{Fe}-\mathrm{Mo} 250$ ), le grossissement du grain peut intervenir car la précipitation intergranulaire diminue la quantité d'éléments ségrégés dans les portions de joints entre carbures ce qui, simultanément, déstabilise les dislocations extrinsèques et permet leurs réactions. On parle de migration induite par la précipitation.

- Lorsqu'une ségrégation (active ou non) ou une fine précipitation intervient, la taille des grains diminue ( $<50 \mu \mathrm{m}$ ), les profils des joints sont hors d'équilibre. C'est le cas des alliages $\mathrm{Fe}-4 \% \mathrm{Ni}$ et $\mathrm{Fe}-\mathrm{Cr}$ à 90 et $150 \times 10^{-4} \mathrm{C}$ pour lesquels les pourcentages de joints spéciaux sont voisins de $25 \%$. On peut supposer que lors de la recristallisation primaire des alliages $\mathrm{Fe}-\mathrm{Ni}$, le carbone réparti dans les interfaces abaisse leur énergie; l'équilibre correspondant à l'énergie 
minimum est alors atteint pour des positions différentes de celles prévues géométriquement [6].

Dans le cas d'une ségrégation active, il faut tenir compte de la présence des dislocations extrinsèques ancrées par les impuretés pour expliquer la faible mobilité des joints, d'où leur difficulté d'atteindre des positions d'équilibre et la faible proportion de joints spéciaux.

5. Conclusion. - Cette étude dégage les conditions de stabilité des dislocations extrinsèques dans les joints de grains d'alliages ferreux. La présence de ces défauts semble nécessiter l'intervention dans l'inter- face d'une ségrégation «active» présentant deux critères essentiels :

- une forte affinité chimique entre les éléments, interstitiel et substitutionnel ségrégés,

— un facteur de taille favorable entre ces éléments et le fer.

Dans ce cas, les défauts peuvent ultérieurement servir de sites de nucléation de précipités. La précipitation entraîne la disparition des dislocations extrinsèques.

Sur la base de ces processus il est possible d'analyser la mobilité des joints de grains et d'interpréter les différentes microstructures de recristallisation obtenues pour divers alliages.

\section{Bibliographie}

[1] Priester, L., Revue Phys. Appl. 15 (1980) 789.

[2] Bollmann, W., Crystals Defects and Crystalline Interface (Springer Verlag, N.Y.) 1970.

[3] Brokman, A., Balluffi, R. W., Acta Met. 29 (1981) 1703.

[4] Warrington, D. H., Boon, M., Acta Met. 23 (1975) 599.

[5] Khalfallah, O., thèse, Orsay (1980).

[6] Khalfallah, O., Priester, L., Revue Phys. Appl. 16 (1981) 153-163.

[7] Ralph, B., Ecob, A. J., Porter, C. Y., Deformation of Polycrystals. Mechanisms and Microstructures, Ed. N. Hansen, Riso National Laboratory, 1981, p. 111.

[8] Pond, R. C., Smith, D. A., Philos. Mag. 36 (1977) 353366.

[9] Clark, W. A. T., Smith, D. A., J. Mat. Sci. 14 (1979) 776.

[10] Pumphrey, P. H., Gleiter, H., Philos. Mag. 30 (1974) 593.

[11] Pumphrey, P. H., J. Physique Colloq. 36 (1975) C4-1.

[12] VArin, R. A., Phys. Status Solidi A 52 (1979) 347.

[13] Gleiter, H., Scr. Met. 11 (1977) 305.

[14] Darby, T. P., Schindler, R., Balluffi, R. W., Philos. Mag. A 37 (1978) 245
[15] Guillope, M., Poirier, J. P., Acta Met. 28 (1980) 163.

[16] Dingley, D. J., Pond, R. C., Acta Met. 27 (1979) 66.

[17] Varin, R. A., J. Mat. Sci. 14 (1979) 811.

[18] Adamson, J. P., Martin, J. W., Acta Met. 19 (1971) 1015.

[19] Buttler, E. P., Swann, P. R., Acta Met. 24 (1976) 343.

[20] Jones, A. R., Howell, P. R., RalPh, B., J. Mat. Sci. 11 (1976) 1595.

[21] Howell, P. R., Jones, A. R., Ralph, B., J. Mat. Sci. 10 (1975) 1351.

[22] Bouchet, D., Priester, L., J. Mat. Sci. 14 (1979) 2205.

[23] Guyot, P., Simon, J. P., J. Physique Colloq. 36 (1975) C4-141.

[24] Mclellan, R. B., Farraro, R., Acta Met. 25 (1977) 1217.

[25] Forest, B., Thèse, Grenoble (1978).

[26] Russel, K. C., Aaronson, H. I., Scr. Met. 8 (1974) 559.

[27] Unwin, P. N. T., Nicholson, R. B., Acta Met. 17 (1969) 1379.

[28] Bouzaher, A., Thèse, I.N.P. Grenoble (1981).

[29] Hondros, E. D., J. Physique Colloq. 36 (1975) C4-116.

[30] ORABI, G., Thèse docteur-Ingénieur, Orsay (1978). 